\title{
Twenty-Four-Hour Profiles and Pulsatile Patterns of Insulin Secretion in Normal and Obese Subjects
}

\author{
K. S. Polonsky, B. D. Given, and E. Van Cauter \\ The University of Chicago, Pritzker School of Medicine, Chicago, Illinois 60637; and The Institute of Interdisciplinary Research, \\ Free University of Brussels, B-1070 Belgium
}

\section{Abstract}

The pattern of endogenous insulin secretion over a 24-h period, which included three mixed meals, was evaluated in 14 normal volunteers and 15 obese subjects. Insulin secretory rates were calculated from plasma $C$-peptide levels using individually derived C-peptide kinetic parameters and a validated open twocompartment model of peripheral C-peptide kinetics. Insulin secretion rates were consistently elevated in the obese subjects under basal conditions (11.6 \pm 1.2 vs. $5.4 \pm 0.5 \mathrm{nmol} / \mathrm{h})$ and in the $4 \mathrm{~h}$ after breakfast $(139 \pm 15$ vs. $63 \pm 5 \mathrm{nmol} / 4 \mathrm{~h}, P$ $<0.001$ ), lunch (152 \pm 16 vs. $67 \pm 5 \mathrm{nmol} / 4 \mathrm{~h}, P<0.001)$, and dinner (145 \pm 18 vs. $65 \pm 6 \mathrm{nmol} / 4 \mathrm{~h}, P<0.001)$.

In the normal subjects, basal insulin secretion represented $50 \pm 2.1 \%$ of total $24-h$ insulin production, insulin secretion returned to baseline between meals, and equal quantities of insulin were secreted in the $4 \mathrm{~h}$ after breakfast, lunch, and dinner, despite the fact that subjects consumed half the number of calories at breakfast compared to lunch and dinner. Overall glucose responses were also similar after the three meals.

In contrast, the pattern of insulin secretion in obese subjects was largely normal, albeit set at a higher level. However, the insulin secretion rate after meals did not return to baseline, and the secretion rate immediately before lunch $(350.5 \pm 81.9$ $\mathrm{pmol} / \mathrm{min})$ and dinner $(373.6 \pm 64.8 \mathrm{pmol} / \mathrm{min})$ was considerably higher than the secretion rate immediately before breakfast (175.5 $\pm 18.5 \mathrm{pmol} / \mathrm{min})$. In these overweight subjects, the glucose response after lunch was lower than after dinner.

Analysis of individual 24-h insulin secretory profiles in the normal subjects revealed that insulin secretion was pulsatile. On average 11.1 \pm 0.5 pulses were produced in each $24-\mathrm{h}$ period. The most prevalent temporal distribution of postmeal secretory pulses was two pulses after breakfast and three pulses after both lunch and dinner. Insulin secretion was also pulsatile during the period without meal stimuli: $3.9 \pm 0.3$ pulses occurred during the period of overnight sampling and in the 3-h period before ingestion of the breakfast meal. In the obese subjects, the number and timing of secretory pulses was similar to those of normal volunteers, although the amplitude of the pulses was significantly greater. In both groups of subjects, $>\mathbf{8 0} \%$ of insulin pulses were concomitant with a pulse in glucose concentration in the postmeal period. The concomi-

Address correspondence to Dr. Polonsky, University of Chicago, Dept. of Medicine, Box 435, 5841 S. Maryland Ave., Chicago, IL 60637.

Received for publication 6 April 1987 and in revised form 31 July 1987.

J. Clin. Invest.

(c) The American Society for Clinical Investigation, Inc.

0021-9738/88/02/0442/07 \$2.00

Volume 81, February 1988, 442-448 tancy rate was significantly lower in the interval without the meal stimuli, averaging $47 \%$ in both groups.

Thus in obesity, although hypersecretion of insulin can be documented, the temporal pattern of secretion is largely unaltered, which suggests that the functioning beta cell mass is enhanced, but normal regulatory mechanisms influencing secretion are still operative.

\section{Introduction}

In recent years, a procedure that allows insulin secretion rates to be estimated from peripheral C-peptide concentrations, using a 2-compartment mathematical model for C-peptide distribution and metabolism, has been developed and validated (1-3). This approach, termed "deconvolution," provides a unique tool for investigating the dynamics of insulin secretion under non-steady state conditions. Indeed, the normal pattern of insulin secretion during ingestion of a mixed diet has not yet been defined. The possible existence of alterations of this pattern in obesity and other pathological states remains to be investigated.

In the present study, the application of deconvolution, together with rapid blood sampling, provided the first detailed description of the 24-h pattern of pancreatic insulin secretion during ingestion of a mixed diet in normal and obese subjects.

\section{Methods}

\section{Subjects}

Studies were performed in the 15 obese subjects and 14 age- and sex-matched volunteers whose demographic data are presented in detail in the companion manuscript (4).

\section{Experimental protocols}

The data to be described in the present manuscript represent an analysis performed on the insulin, C-peptide, and glucose concentrations and insulin secretion rates measured at frequent intervals during a 24-h period. After a 10-h overnight fast, samples for insulin, C-peptide, and glucose were drawn at 15-30-min intervals for a total of $24 \mathrm{~h}$ beginning at 0600 . During the course of the investigation, subjects consumed a standard diet of $30 \mathrm{cal} / \mathrm{kg}$, comprised of $50 \%$ carbohydrate, $15 \%$ protein, and $35 \%$ fat. $20 \%$ of total calories were eaten at breakfast and $40 \%$ with lunch and dinner, respectively. The three meals were consumed at 0900,1300 , and 1800 and subjects were required to complete the meal within $30 \mathrm{~min}$. Details of the sampling techniques and assay procedures have been described previously and are provided in the companion manuscript and elsewhere (4-6).

\section{Data analysis}

For each subject, the insulin secretion rate during each sampling interval was derived from the profile of plasma C-peptide levels as previously described $(1,2)$.

Calculation of basal glucose levels and insulin secretion rates. The basal glucose level and the basal insulin secretory rate were estimated 
as the mean of the corresponding values obtained between 0600 and 0900 , i.e., during the 3-h interval preceding breakfast. The basal, i.e., non-meal stimulated, insulin secretion over the 24-h span was deduced by extrapolating the basal secretory rate over the 24-h period.

Calculation of 24-h insulin secretion and postmeal responses. The total amount of insulin secreted over the 24-h span was estimated as the area under the 24-h profile of secretory rates. The total amount of insulin secreted after each meal was calculated as the area under the 4-h interval after meal ingestion. To evaluate possible differences in the dynamics of insulin secretion after breakfast, lunch, and dinner, respectively, the area under the curve was also calculated for each hour after the meal.

The glucose response to each meal was similarly calculated as the area under the curve during the 4-h interval after meal time. The dynamics of the postmeal glucose response were also estimated by calculating the area under the glucose curve for each hour after meal time.

Pulse analysis of plasma profiles of C-peptide, insulin, and glucose. Each individual 24-h profile of plasma insulin, glucose, and C-peptide was analyzed using ULTRA, an algorithm for pulse identification that has been applied to the analysis of episodic secretion in a number of hormonal systems (7-12). The general principle of the algorithm is the elimination of all peaks of plasma concentration for which either the increment (difference between the peak value and the preceding nadir) or the decrement (difference between the peak value and the next nadir) does not exceed a certain threshold related to measurement error. The peaks that do not meet these threshold criteria are eliminated from the data set using an iterative process, leaving a "clean" series in which all remaining peaks are assumed to represent significant pulses.

Measurement error in determining the concentration of a blood constituent is primarily determined by the precision of the assay involved. In the present study, the intraassay coefficient of variation for both the insulin and C-peptide assays was 4\%, and the intraassay coefficient of variation of the glucose measurements was $3 \%$. The threshold was set at twice the measurement error, i.e., $8 \%$ for insulin and C-peptide and $6 \%$ for glucose.

Each pulse in the clean series was characterized in terms of total duration, duration of the ascending and declining limbs, and absolute increment (difference between level at the peak and level at the preceding trough). The clean, rather than original, C-peptide series were used to calculate the insulin secretory rates to avoid amplification of noise involved in the deconvolution process.

Pulse analysis of the 24-h profiles of insulin secretory rates. Peaks of insulin secretory rates were considered as true secretory pulses if they met at least one of the following conditions:

(a) Both the increment and the decrement exceeded, in relative terms, $12 \%$, i.e., three times the measurement error for $\mathrm{C}$-peptide, a conservative threshold selected to exclude possible noise. This criterion for the significance of individual pulses is based on the assumption that pulses of relative amplitude greater than the above levels could not have been due to random variations related to measurement errors.

(b) The maximum level of the pulse occurred within $15 \mathrm{~min}$ of the maximum level of a peak in either serum insulin or glucose concentrations. Since C-peptide and insulin are co-secreted in equal concentration from the beta cell, the concomitance of peaks of secretory rate with peaks of serum insulin and/or plasma glucose provided physiological evidence that the increase in secretion represented a true secretory pulse.

Statistical tests. The significance of group differences was evaluated by the $t$ test for unpaired data. Where appropriate, multiple variables were compared by multivariate analysis of variance. The temporal distribution of pulses was compared in the two groups by frequency table analysis. The statistical significance of differences was evaluated at the 5\% level. Unless otherwise stated all group data are expressed as mean \pm SEM.

\section{Results}

\section{Insulin, C-peptide, and glucose concentrations}

The mean profiles of plasma insulin, C-peptide, and glucose measured over the 24-h period on a mixed diet are shown in Fig. 1. On the average, glucose concentrations were not significantly different in the obese subjects (obese vs. normals, $108.0 \pm 1.8$ vs. $109.9 \pm 1.9 \mathrm{mg} / \mathrm{dl}, P<0.5)$. Insulin $(24.1 \pm 2.1$ vs. $62.9 \pm 10.7 \mu \mathrm{U} / \mathrm{ml}, P<0.003)$ and C-peptide concentrations $(0.91 \pm 0.09$ vs. $1.58 \pm 1.07 \mathrm{pmol} / \mathrm{ml}, P<0.001)$ were significantly greater in the obese group.

Patterns of postmeal glucose responses. The postmeal glu-
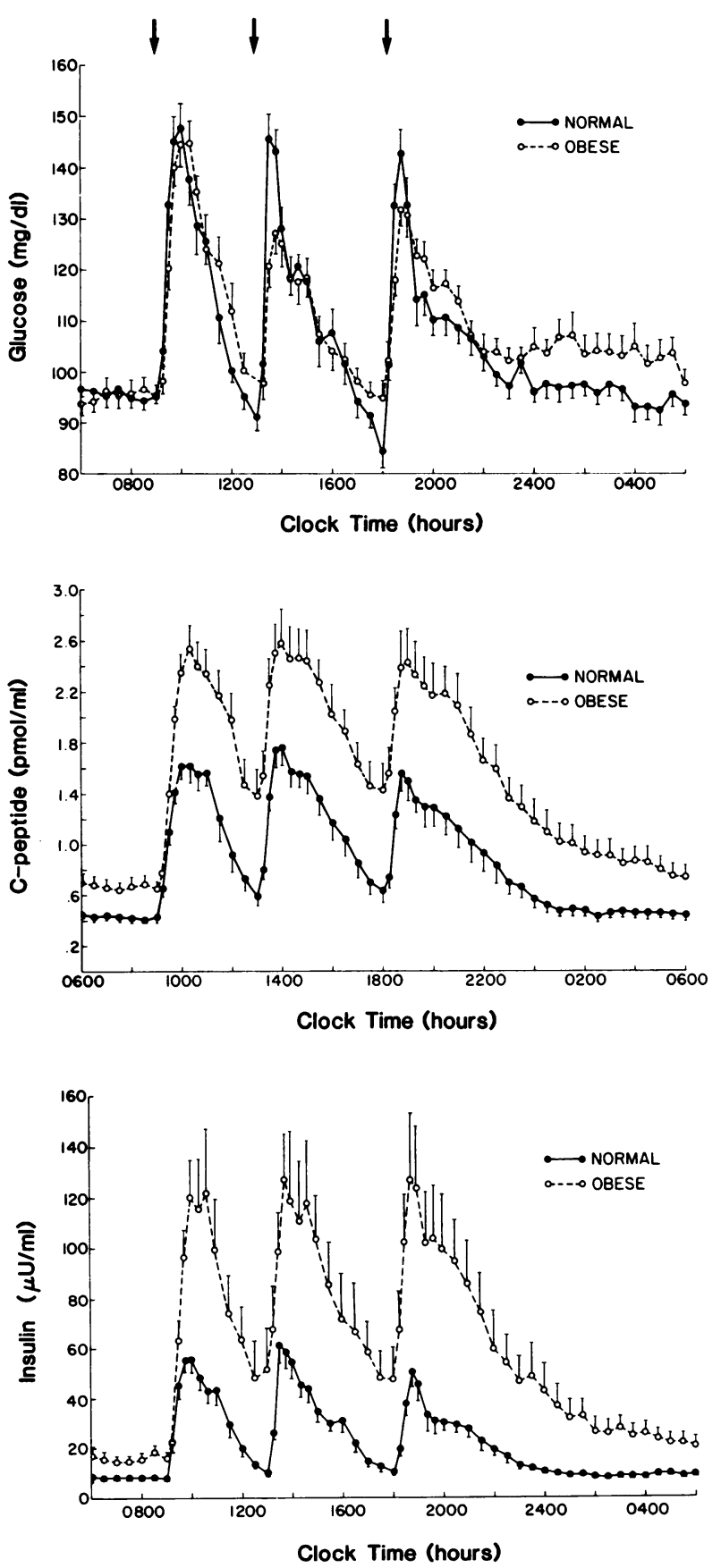

Figure 1. Mean 24-h profiles of plasma concentrations of glucose, Cpeptide, and insulin in the normal and obese subjects. 
cose responses were compared and the total areas under the glucose curves during the 4 -h period after meal time were found to be similar for breakfast, lunch, and dinner in normal subjects. In contrast, in obese patients, the postmeal glucose response was significantly smaller after lunch than after breakfast $(3,852 \pm 759 \mu \mathrm{g} / \mathrm{dl}$ per min after lunch vs. 5,892 \pm 601 $\mu \mathrm{g} / \mathrm{dl}$ per min after breakfast, $P<0.03$ ). The magnitude of the postdinner response was intermediate between that of the postbreakfast and the postlunch response, without differing significantly from either.

The temporal pattern of meal-induced glucose elevations was investigated by calculating the area under the glucose curve above the premeal level before and for each hour after breakfast, lunch, and dinner for a total of $4 \mathrm{~h}$ after each meal. In normal volunteers, the dynamics of the glucose response appeared to parallel those of insulin secretion in that the response after dinner was more prolonged than after breakfast and lunch. Indeed, after breakfast, the glucose increment in the $2 \mathrm{~h}$ after meal time corresponded to $79.2 \pm 2.5 \%$ of the 4-h response, while after lunch and dinner, these percentages fell to $74.0 \pm 3.5$ and $62.6 \pm 2.8 \%$. The differences between dinner and breakfast $(P<0.001)$ and dinner and lunch were statistically significant $(P<0.02)$. In contrast, in obese subjects, differences in postmeal glucose dynamics between breakfast, lunch, and dinner did not reach statistical significance. In the obese subjects, the ratio of the area under the glucose curve to the area under the insulin secretory rate curve was $\sim 50 \%$ of the mean ratio calculated in normal subjects, a result consistent with the well-known insulin resistance in obesity.

Estimation of basal and meal-stimulated insulin secretion rates. The mean profiles of insulin secretory rates for normal and obese subjects are shown in the upper panel of Fig. 2. Insulin secretion rates were elevated in the obese group both under basal fasting conditions $(94.9 \pm 10.8$ vs. $189.0 \pm 19.9$ $\mathrm{pmol} / \mathrm{min}, P<0.001$ ) and in response to meals. When the basal insulin secretion rate was extrapolated over a $24-\mathrm{h}$ period and expressed as a percentage of the total 24-h insulin secretion, basal secretion represented $50.1 \pm 3.1 \%$ of the total $24-\mathrm{h}$ insulin secretion in normal subjects and $45.2 \pm 2.2 \%$ in obese patients $(P<0.2)$. The relationship between basal and mealstimulated insulin secretion was explored by expressing, in each individual, the insulin secretion rate at each sampling time as a percentage of the basal insulin secretion rate. The results of this analysis are in the lower panel of Fig. 2. When the secretion rate is expressed as a function of basal secretion, the curves for the normal and obese subjects are virtually superimposable, except that insulin secretion fails to return to baseline between meals in the obese subjects. Thus, immediately preceding ingestion of the breakfast, lunch, and dinner meals, insulin secretion rates were $100.3 \pm 11.5,94.1 \pm 13.4$, and $118.4 \pm 15.6 \mathrm{pmol} / \mathrm{min}$ in the normal subjects. In contrast, in the obese, the insulin secretion rates immediately before lunch $(350.5 \pm 81.9 \mathrm{pmol} / \mathrm{min})$ and dinner $(373.6 \pm 64.8 \mathrm{pmol} /$ min) were significantly higher than the secretion rate immediately before breakfast $(175.5 \pm 18.5 \mathrm{pmol} / \mathrm{min}, P<0.05)$.

Although subjects consumed $20 \%$ of total calories with breakfast and $40 \%$ with lunch and dinner, respectively, similar quantities of insulin were secreted in response to each of the three meals (Fig. 2). Indeed, the normal subjects secreted $63.1 \pm 5.0,67.5 \pm 4.7$, and $64.7 \pm 5.9 \mathrm{nmol}$ insulin in the $4 \mathrm{~h}$ immediately after breakfast, lunch, and dinner, respectively. Corresponding values in the obese subjects were $138.3 \pm 14.9$,
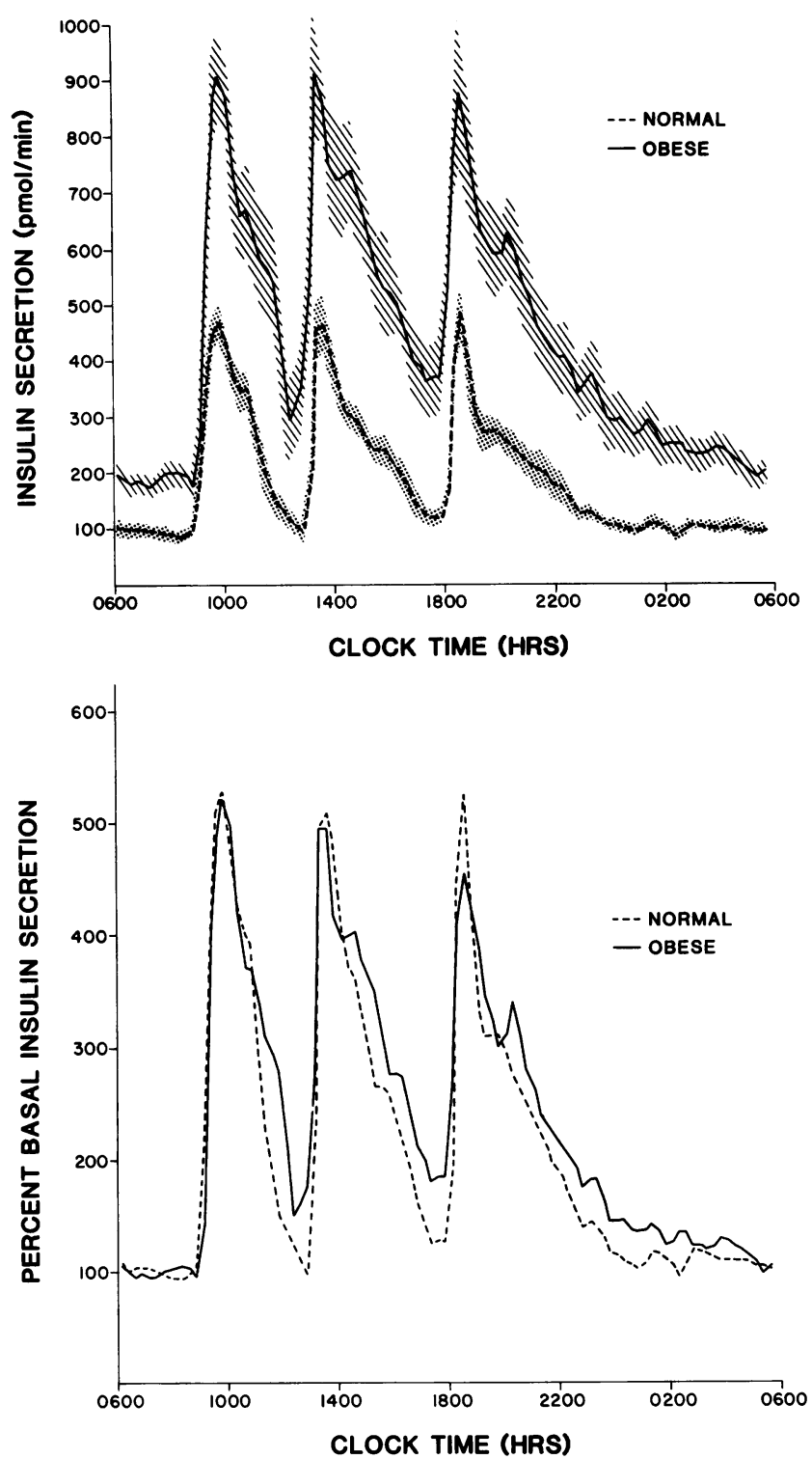

Figure 2. Mean 24-h profiles of insulin secretion rates in the normal and obese subjects (top). The hatched areas represent \pm 1 standard error of the mean. The curves in the lower panel were derived by dividing the insulin secretion rate measured in each subject by the basal secretion rate derived in the same subject. Mean data for the normal (-- ) and obese $(-)$ subjects are shown.

$152.2 \pm 16.3$, and $145.2 \pm 18.1 \mathrm{nmol}$. The total amount of insulin secreted after each of the meals did not differ in either of the two groups, although meal responses were significantly greater than normal in the obese subjects.

Patterns of postmeal insulin secretion rates. The pattern of insulin secretion in response to meals was further investigated by calculating the amount of insulin secreted in the hour before and in each hour after breakfast, lunch, and dinner for a duration of $4 \mathrm{~h}$. After breakfast, in normal subjects, $71.6 \pm 1.6 \%$ of the total 4-h secretory response to breakfast was released in the first $2 \mathrm{~h}$ after the meal, and $28.4 \pm 1.6 \%$ was secreted in the 3rd and 4th hours. In response to lunch and dinner, insulin secretion did not fall as rapidly as it did after breakfast. Thus, in the first $2 \mathrm{~h}$ after each of these meals $62.8 \pm 1.6$ and $59.6 \pm 1.4 \%$ of the $4-h$ insulin secretory response was produced, with $37.2 \pm 1.6$ and $40.4 \pm 1.4 \%$ in the 3rd and 4th hours, re- 
spectively. The differences between breakfast and both lunch and dinner were statistically significant $(P<0.0001)$ Thus, insulin secretion fell more rapidly after breakfast than after lunch or dinner. The observed difference in secretory dynamics, however, may be related to the fact that the breakfast meal was smaller than the other two meals, although it should be noted that the total insulin secretory response was similar to that of lunch and dinner.

The obese subjects also secreted more insulin in the 1 st and 2nd than in the 3rd and 4th hours after each meal, but insulin secretion did not fall more rapidly after breakfast than lunch and dinner in these subjects. Indeed, in the first $2 \mathrm{~h}$ after breakfast, lunch, and dinner, the obese subjects produced $62.8 \pm 2.5,59.0 \pm 1.2$, and $57.2 \pm 0.9 \%$ of the $4-\mathrm{h}$ meal secretory response.

Identification and analysis of insulin secretory pulses. Representative 24-h profiles of insulin secretion from two normal and two obese subjects are shown in Fig. 3. The pulsatile nature of insulin secretion in response to the meals as well as during overnight sampling is visually apparent. Due to individual differences in the precise timing of the pulses, pulses are not seen in the mean profiles shown for each group in Fig. 2.

Each 24-h profile was submitted to formal pulse analysis to identify the statistically significant pulses of insulin secretion.
Fig. 4 summarizes, for each group, the temporal distribution and mean increment of pulses identified in the 4-h interval between breakfast and lunch, in the 5-h interval between lunch and dinner, and in the 5-h interval immediately after dinner. Pulses were classified according to their times of occurrence after meal presentation. In the diagrams shown in Fig. 4, the width of the vertical bars represents the mean \pm SD of the times of occurrence of pulses in relation to the meal and their height illustrates the mean pulse increment above the level of the preceding nadir.

After breakfast, $1.8 \pm 0.2$ and $1.9 \pm 0.2$ secretory pulses were identified in the normal volunteers and obese subjects, respectively. Within the 1st hour after breakfast, a significant secretory pulse was identified in all 14 normal volunteers and in 14 of the 15 obese subjects. The peaks of these secretory pulses occurred $42.8 \pm 3.4$ and $49.3 \pm 2.3 \mathrm{~min}$ after the meal in the two groups, respectively $(P<0.1)$, and represented increments in secretion of $442.4 \pm 20.6$ and $816.6 \pm 67.8 \mathrm{pmol} / \mathrm{min}(P$ $<0.001$ ). 7 of the 14 normal controls and 8 of the 15 obese subjects had a second secretory pulse which began in the 2 nd hour after breakfast. The timing of this secondary postbreakfast peak did not differ between normal and obese subjects but its mean increment tended to be larger in obese patients $(233.9 \pm 66.0 \mathrm{pmol} / \mathrm{min}$ vs. $86.4 \pm 14.4 \mathrm{pmol} / \mathrm{min}, P<0.06)$. In
NORMAL
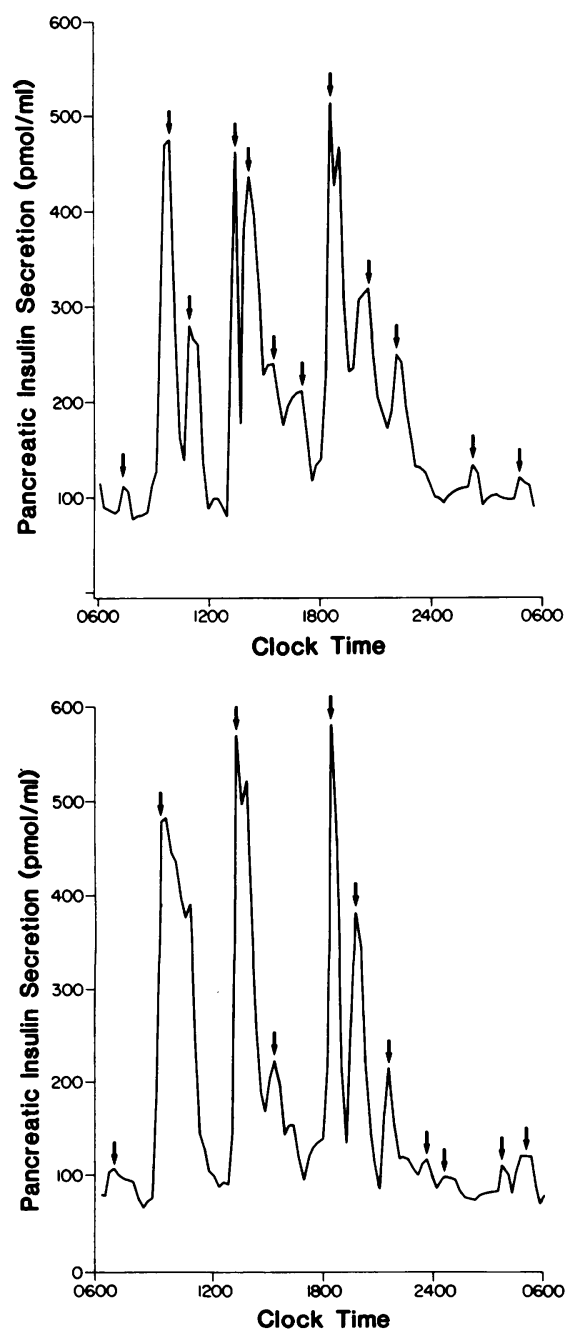

OBESE
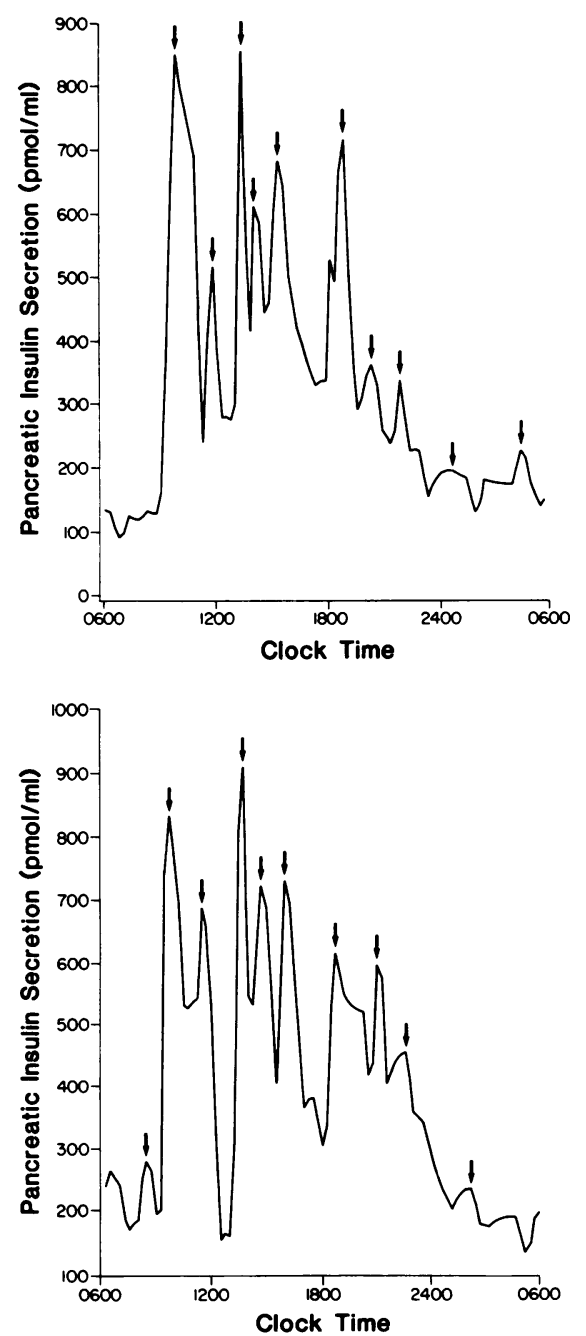

Figure 3. Patterns of insulin secretion in normal and obese subjects. Four representative 24-h insulin secretory profiles from two normal weight subjects (left) and two obese subjects (right). Meals were consumed at 0900,1300 , and 1800 . Statistically significant pulses of secretion are shown by the arrows. 

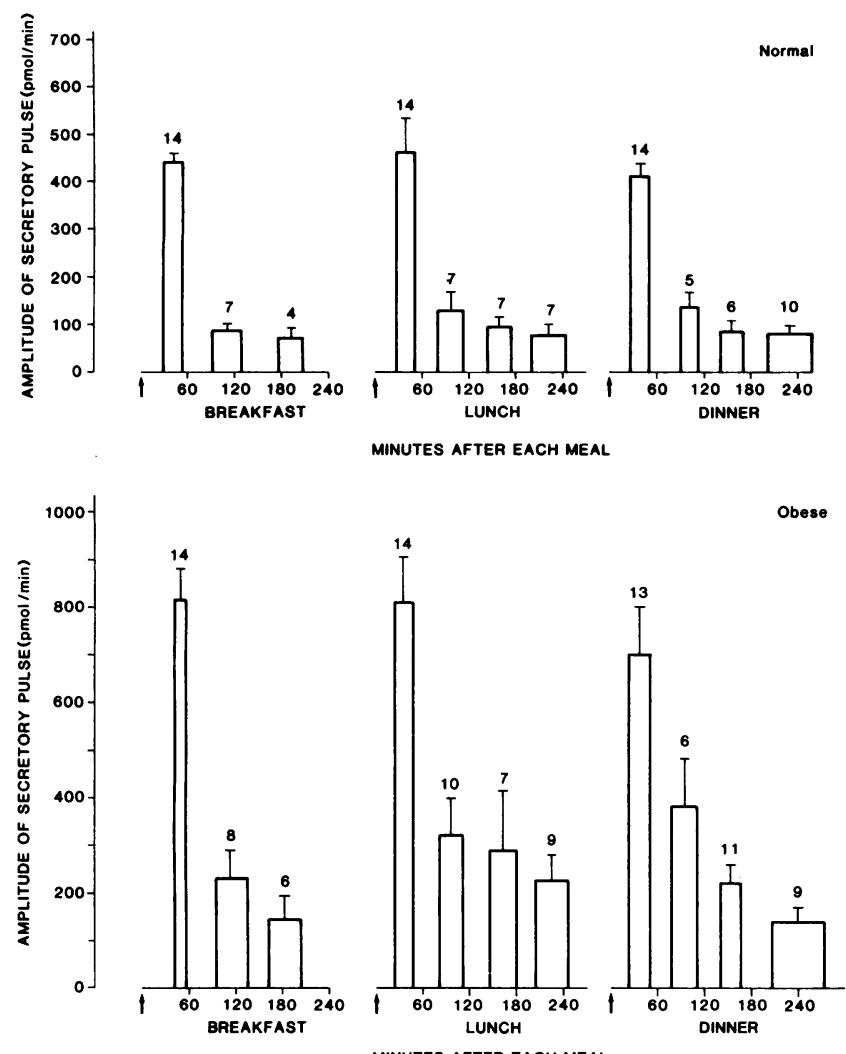

MINUTES AFTER EACH MEAL

ㅁormal
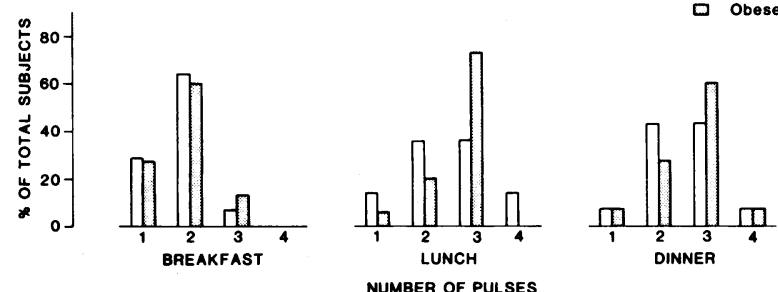

NUMBER OF PULSES

Figure 4. Temporal distribution and amplitude of insulin secretory pulses after breakfast, lunch, and dinner in the normal (top) and obese (middle) subjects. The increase in secretion over the preceding nadir is represented on the vertical axis. The timing of pulses in relation to the meal is represented by the position of the vertical bars on the horizontal axis. The center of each vertical bar represents the average time of peak secretion in pulses that began within the 1st, 2nd, 3rd, or 4th and 5th hours after lunch and dinner. The width of each vertical bar represents \pm 1 standard deviation of the average time at which peak secretion in the corresponding pulse occurred. The numbers above each vertical bar refer to the number of subjects in each group in whom secretory pulses were identified at the corresponding times. The data in the bottom panel depicts the percentage of subjects in each group in whom one, two, three, or four pulses of secretion were identified after each meal.

4 of the 14 normal volunteers and in 6 of the 15 obese subjects, an additional significant secretory pulse beginning in the 3rd hour after breakfast was detected. Differences between normal and obese subjects in either timing or amplitude of these additional postbreakfast secretory pulses were not statistically significant.

As shown in Fig. 4, multiple insulin secretory pulses were also identified after lunch and dinner. After these meals, up to four pulses of insulin secretion were observed in both groups of subjects. In the 5-h time interval between lunch and dinner, an average of $2.5 \pm 0.3$ and $2.7 \pm 0.2$ secretory pulses were identified in the normal and obese subjects, respectively. In the $5 \mathrm{~h}$ after dinner, $2.6 \pm 0.2$ and $2.7 \pm 0.2$ pulses were observed in the two groups, respectively. In the obese subjects, the times of occurrence of the pulses were not significantly different from normal, but as shown in Fig. 4, the pulses were significantly larger by multivariate analysis of variance (Hotelling-Lawley Trace statistic, $P<0.003$ ).

The frequency distribution of the pulses that occurred in relation to meals is shown in the lower panel of Fig. 4. After breakfast, the most prevalent pattern was that of two postmeal pulses in both groups of subjects. After lunch and dinner, the most prevalent pattern was that of three postmeal pulses in both normal and obese subjects. After breakfast, $86 \%$ of the subjects had no more than two secretory pulses. After either lunch or dinner, only $50 \%$ of the subjects had two pulses or less. The differences in the postmeal response after breakfast, on the one hand, and lunch and dinner, on the other hand, were statistically significant (Chi square test, $P<0.05$ ).

Pulses of insulin secretion that did not appear to be meal related were also identified. Between 2300 and 0600 and in the $3 \mathrm{~h}$ before breakfast ( 0600 to 0900$)$, on average $3.9 \pm 0.3$ secretory pulses were present in both the normal and obese subjects. During this period as well, pulse amplitude was significantly greater in the obese $(85 \pm 14 \mathrm{pmol} / \mathrm{min})$ than in the normal subjects $(31 \pm 24 \mathrm{pmol} / \mathrm{min}, P<0.001)$. Thus, over the $24-\mathrm{h}$ period of observation, a total of $11.1 \pm 0.5$ pulses were identified in the normal subjects and $11.9 \pm 0.6$ in the obese group. The mean time of recurrence of insulin pulses appeared to be shorter during the 14-h postmeal period (normals, $123 \pm 9 \mathrm{~min}$; obese, $110 \pm 7 \mathrm{~min}$ ) than during the 10 -h interval without secretory stimuli (normals, $172 \pm 17 \mathrm{~min}, P<0.01$; obese, $170 \pm 14 \mathrm{~min}, P<0.005$ ).

In the normal subjects, $87 \pm 3 \%$ of postmeal pulses in insulin secretion but only $47 \pm 8 \%$ of non-meal related pulses were concomitant with a pulse in glucose. Similar values of $81 \pm 4$ and $47 \pm 6 \%$ were observed in the obese subjects. Differences between post-meal and non-meal-related pulses were highly significant $(P<0.001)$.

\section{Discussion}

Recently, biosynthetic human C-peptide, identical in structure to pancreatic human $\mathrm{C}$-peptide, has become available for use in human studies (2). We have demonstrated that if the kinetics of C-peptide are defined in individual subjects by analyzing the decay curve after a bolus injection of exogenous C-peptide, the pancreatic insulin secretion rate can be accurately derived from peripheral C-peptide concentrations using an open 2-compartment mathematical model (2). In the present study, we have used this technique to define in detail the dynamics of insulin secretion after a mixed meal at breakfast, lunch, and dinner in normal and obese subjects.

This approach has allowed a number of important quantitative and qualitative aspects of insulin secretion to be defined. First, insulin secretion is oscillatory, with pulses of secretion being detected both in response to meals and in the absence of secretory stimuli. In normal subjects, the most prevalent pattern of postmeal insulin secretion consisted of a damped oscillation with two pulses after breakfast, and three pulses after both lunch and dinner. Insulin pulses were less frequent and occurred less regularly in the absence of secretory stimuli. 
These postmeal oscillations could reflect an ongoing oscillation of basal secretion, which is amplified by meal stimulation. Differences in pulse frequency between the postmeal period and the time interval without secretory stimuli would then reflect our inability to detect very small secretory pulses in the basal period. Evidence supporting this view has been recently provided by a study showing large sustained oscillations of plasma insulin in normal men during continuous enteral nutrition (13). The biphasic patterns of plasma insulin, C-peptide, and glucose levels observed by Robbins et al. (12) in response to an early lunch (11:30) of mixed composition given to normal volunteers probably also reflect an oscillatory secretory response. Thus, a complex frequency spectrum appears to characterize beta cell function, including the well-known rapid pulsations in the 10-14-min range (14-16) and the slower oscillations with ultradian periods in the range of $1-3 \mathrm{~h}$ presented here.

Synchronous glucose oscillations were clearly associated with postmeal pulses of insulin secretion. The much lower concomitancy rate observed under nonstimulated conditions could reflect random associations, or, again, the limit of sensitivity of the pulse identification technique. At any rate, in this as well as in other studies $(12,14,16)$, the close temporal association between pulses of insulin secretion and of blood glucose levels clearly indicates that the postmeal oscillations are characteristic of the insulin-glucose regulatory mechanisms.

The present study has also provided evidence that the beta cell response to mixed meal ingestion may be influenced by the time of day when the meal is presented. Thus, the same quantity of insulin was secreted in response to breakfast, lunch, and dinner despite the fact that half the number of calories were consumed at breakfast as at the other two meals. Studies are currently under way in our laboratory to determine if the greater insulin secretory response in relation to the number of calories after breakfast is due to a circadian variation in the responsiveness of the beta cell to meal ingestion, or to diurnal differences in insulin sensitivity, or whether it relates to the longer period of fasting before breakfast.

Under basal conditions and in response to meals, insulin secretion rates are increased in obesity. However, the pattern of insulin secretion was found to be qualitatively normal in a number of important respects. Thus, basal insulin secretion represented $\sim 50 \%$ of 24 -h insulin production in both obese and normal subjects. Note that these relative proportions of basal and postmeal secretion are in close agreement with empiric observations using either the artificial endocrine pancreas, or continuous subcutaneous insulin infusion via the insulin pump, which have found that the delivery of $\sim 50 \%$ of the total daily insulin dose as a constant basal infusion and $\sim 50 \%$ as premeal boluses, most closely approximates the normal situation (17-20). The persistence of a normal relationship between basal and meal-stimulated insulin secretion in obesity was clearly apparent when the secretion rates were expressed as a percentage of the basal secretion. This procedure yielded 24-h patterns of insulin secretion that were virtually superimposable (Fig. 2, bottom), with the exception that insulin secretion failed to return to baseline between meals in the obese subjects. These findings suggest that, in obesity, the increase in insulin secretion is not due to a hyperresponsiveness to secretory stimuli.

Finally, the temporal organization of insulin secretory pulses and their association with fluctuations of blood glucose were, in every respect, similar in obesity, with the only consistent difference being that the pulses were larger than in normals. Taken together, these observations indicate that insulin secretion is normally pulsatile under basal conditions and after meals. In obesity, the dynamics of insulin secretion are largely unaltered, which suggests that the observed increase in secretion rates results from excessive insulin output from an abnormally large functional beta cell mass, but that normal regulatory mechanisms are still operative.

\section{Acknowledgments}

Dr. Polonsky is the recipient of a Research Career Development Award from National Institutes of Health (NIH) and a Career Development Award from the Schweppe Foundation. The authors wish to thank Virginia Kuvakos for expert secretarial assistance. Dr. Arthur Rubenstein has provided invaluable support and guidance in the design and interpretation of the studies described in this manuscript.

This work was supported in part by grant DK-31842 from NIH, the Diabetes Research and Training Center (DK-20595), and the Clinical Research Center at the University of Chicago (RR-00055), and by grants from Eli Lilly \& Co., the Belgian Ministere de la Politique Scientifique (Actions Concertees), and Fonds National de la Recherche Scientifique Medicale.

\section{References}

1. Eaton, R. P., R. C. Allen, D. S. Schade, K. M. Erickson, and J. Standefer. 1980. Prehepatic insulin production in man: kinetic analysis using peripheral connecting peptide behavior. J. Clin. Endocrinol. Metab. 51:520-528.

2. Polonsky, K. S., J. Licinio-Paixao, B. D. Given, W. Pugh, P. Rue, J. Galloway, T. Karrison, and B. Frank. 1986. Use of biosynthetic human C-peptide in the measurement of insulin secretion rates in normal volunteers and type I diabetic patients. J. Clin. Invest. 77:98105.

3. Polonsky, K. S., B. Frank, W. Pugh, A. Addis, T. Karrison, P. Meier, H. Tager, and A. H. Rubenstein. 1986. The limitations and valid use of C-peptide as a marker of the secretion of insulin. Diabetes. 35:370-386.

4. Polonsky, K. S., B. D. Given, L. Hirsch, E. T. Shapiro, H. Tillil, C. Beebe, J. A. Galloway, B. H. Frank, E. Van Cauter, and T. Karrison. A quantitative study of insulin secretion and clearance in normal and obese subjects. 81:435-441.

5. Morgan, C. R., and A. Lazarow. 1963. Immunoassay of insulin: two antibody systems. Plasma insulin levels of normal subdiabetic and diabetic rats. Diabetes. 12:115-126.

6. Faber, O. K., C. Binder, L. Markussen, L. G. Heding, V. K. Naithani, H. Kuzuya, P. M. Blix, D. L. Horwitz, and A. H. Rubenstein. 1978. Characterization of seven C-peptide antisera. Diabetes. 27:170-177.

7. Van Cauter, E. Quantitative methods for the analysis of circadian and episodic hormone fluctuations In Human Pituitary Hormones: Circadian and Episodic Variations. E. Van Cauter and G. Copinschi, editors. Martinus Nyhoff, The Hague, Netherlands. 1-25.

8. Van Cauter, E., M. L'Hermite, G. Copinschi, S. Refetoff, D. Desir, and C. Robyn. 1981. Quantitative analysis of spontaneous variations of plasma prolactin in normal man. Am. J. Physiol. 241:E355E363.

9. Golstein, J., E. Van Cauter, D. Desir, P. Noel, J. P. Spire, S. Refetoff, and G. Copinschi. 1983. Effects of "jet lag" in hormonal patterns. IV time shifts increase growth hormone release. J. Clin. Endocrinol. Metab. 56:433-440.

10. Linkowski, R., J. Mendlewicz, J. Golstein, G. Copinschi, R. Leclercq, M. Brasseur, and E. Van Cauter. 1985. The 24-hour profile of ACTH and cortisol in major depressive illness. J. Clin. Endocrinol. Metab. 61:429-438. 
11. Jaspan, J. B., E. Lever, K. S. Polonsky, and E. Van Cauter. 1986. In vivo pulsatility of pancreatic islet peptides. Am. J. Physiol. 251:E215-E226.

12. Robbins, D. C., J. Jaspan, B. Vasquez, and E. Van Cauter. 1987. Biphasic patterns of peripheral insulin and glucose levels following lunch in normal subjects. Diabetes Care. 10:293-299.

13. Simon, C., G. Brandenberger, and M. Follenius. 1987. Ultradian oscillations of plasma glucose, insulin and C-peptide in man during continuous enteral nutrition. J. Clin. Endocrinol. Metab. 64:669-674.

14. Goodner, C. J., B. C. Walike, D. J. Koerker, J. W. Ensinck, A. C. Brown, E. W. Chideck, J. Palmer, and L. Kulnasy. 1977. Insulin glucagon and glucose exhibit synchronous sustained oscillations in fasting monkeys. Science (Wash. DC). 195:177-179.

15. Hansen, B. C., K. C. Jen, S. B. Pek, and R. A. Wolfe. 1982. Rapid oscillations in plasma insulin, glucagon and glucose in obese and normal weight humans. J. Clin. Endocrinol. Metab. 54:785-792.
16. Lang, D. A., D. R. Matthews, J. Peto, and R. C. Turner. 1979. Cyclic oscillations of basal plasma glucose and insulin concentrations in human beings. N. Engl. J. Med. 301:1023-1027.

17. Nathan, D. M., P. Lon, and J. Avruch. 1982. Intensive conventional and pump therapies in adult Type I diabetes. Ann. Intern. Med. 97:31-36.

18. Schiffrin, A. D., M. DesRosiers, A. Hassan, and M. M. Belmonte. 1984. Intensified insulin therapy in the Type I diabetic adolescents: a controlled trial. Diabetes Care. 7:107-113.

19. Rizza, R. A., J. E. Gerich, M. W. Haymond, R. E. Westland, L. D. Hall, A. H. Clemens, and F. J. Service. 1980. Control of blood sugar in insulin-dependent diabetes: comparison of an artificial endocrine pancreas. Continuous subcutaneous insulin infusion, and intensified conventional insulin therapy. N. Engl. J. Med. 303:1313-1318.

20. Santiago, J. V., A. H. Clemens, W. L. Clarke, and D. M. Kipnis. 1979. Closed-loop and open-loop devices for blood glucose control in normal and diabetic subjects. Diabetes. 28:71-81. 